\title{
The difference in ocular lens equivalent dose to ERCP personnel between prone and left lateral decubitus positions: a prospective randomized study
}

(๑) $\odot \ominus$

\author{
Authors \\ Phonthep Angsuwatcharakon ${ }^{1,2}$, Worawarut Janjeurmat ${ }^{1}$, Anchali Krisanachinda ${ }^{3}$, Wiriyaporn Ridtitid ${ }^{1}$, \\ Pradermchai Kongkam¹, Rungsun Rerknimitr ${ }^{1}$
}

Institutions

1 Division of Gastroenterology, Department of Medicine, Chulalongkorn University and King Chulalongkorn Memorial Hospital, Bangkok, Thailand

2 Department of Anatomy, Chulalongkorn University and King Chulalongkorn Memorial Hospital, Bangkok, Thailand

3 Division of Nuclear Medicine, Department of Radiology, Faculty of Medicine Chulalongkorn University and King Chulalongkorn Memorial Hospital, Bangkok, Thailand

submitted 25.8.2017

accepted after revision 5.3.2018

Bibliography

DOI https://doi.org/10.1055/a-0599-5917 |

Endoscopy International Open 2018; 06: E969-E974

(c) Georg Thieme Verlag KG Stuttgart · New York

ISSN 2364-3722

Corresponding author

Rungsun Rerknimitr, MD, FRCP (London), Professor of Medicine, Division of Gastroenterology, Department of Medicine, Faculty of Medicine Chulalongkorn University and King Chulalongkorn Memorial Hospital, 1873 Rama 4 Road, Patumwan, Bangkok 10330, Thailand

Fax: + 6622564356

ercp@live.com

\section{ABSTRACT}

Background and study aims Endoscopic retrograde cholangiopancreatography (ERCP) is commonly performed in a prone or left lateral decubitus (LLD) position. The ocular lens equivalent doses between the two positions may be different because in the LLD position the tube voltage will automatically increase to maintain the image quality, and the increased distance between the image intensifier and the $\mathrm{X}$-ray tube may result in more scattered radiation. We aimed to compare the ocular lens equivalent doses of ERCP personnel between the two different positions.

Patients and methods Fifty-five patients with ERCP indications were randomized to either prone or LLD positions. One patient in an LLD position was excluded due to technical reasons. Indications for ERCP, patients' vertical thicknesses, fluoroscopy parameters, patients' skin dose rates, and the ocular-lens equivalent doses of ERCP personnel were compared.

Results Baseline characteristics were no different except for vertical thickness, which was significantly higher in the LLD group. The ocular lens equivalent doses (prone vs. LLD) of the primary endoscopist (19.2 vs. $30.7 \mu \mathrm{Sv}, P=0.035)$, and the nurse anesthetist ( 17.3 vs. $42.2 \mu \mathrm{Sv}, P=0.002$ ) were significantly lower in the prone group than in the LLD group. The calculated annual number of procedures not to exceed the exposure allowance in prone and LLD positions were 1,042 and 651 procedures for the primary endoscopist and 1,157 and 473 procedures for the nurse anesthetist, respectively.

Conclusions Ocular-radiation exposure to ERCP personnel was one-third lower in the prone than in LLD position. Therefore, more annual ERCPs could be performed by the personnel.

Clinical.Trials.gov

NCT02791659

TRIAL REGISTRATION: Interventional (Clinical Trial)

NCT02791659

at clinicaltrials.gov

\section{Introduction}

Endoscopic retrograde cholangiopancreatography (ERCP) is the standard procedure for treatment of pancreaticobiliary diseases that require use of fluoroscopy. Thus, personnel involved in the ERCP room are at risk for radiation hazards [1]. lonizing radiation from fluoroscopy potentially causes cell injury to various organs (tissue reaction) [2] and increases risk of cancer or genetic defects (stochastic effect) [3-5]. To avoid this adverse 
effect, the "As Low As Reasonably Achievable (ALARA)" principle is recommended for radiation safety $[5,6]$.

Radiation doses to the patient and medical personnel depend on several factors such as fluoroscopic time, thickness of the patient's exposed body, distance between the X-ray tube and personnel, and distance between image intensifier and patient [5, 7-9]. With the automatic exposure control function, the fluoroscopy system will increase the tube voltage in a thicker object, when compared with the thinner object, to maintain the image quality $[10,11]$. The patient's positions during ERCP can be either prone, supine or left lateral decubitus (LLD) depending on the endoscopist's preference [12]. Because the vertical body thicknesses of prone and supine are similar and thinner than the LLD position, the adjusted beam by the fluoroscopic machine on these two positions is speculated to be lower than in the LLD position [11]. Moreover, the increase in distance between the image intensifier and the X-ray tube or patient could result in the radiation being more scattered in a thicker object. We then hypothesized that the scattered radiation to ERCP personnel could be lower in prone and supine positions than in the LLD position. In our experience, performing ERCP in a supine position is more difficult and technically more challenging compared to a prone position $[12,13]$, while the LLD position had a comparable success rate to that of the prone position [14]. We then aimed to compare the radiation exposure in ERCP personnel between patients lying in a prone position and LLD position and chose the ocular lens, which is the most susceptible organ, as our target of comparison [2].

\section{Patients and methods}

\section{Patients}

This was a parallel prospective randomized study performed at the Excellence Center for Gastrointestinal Endoscopy of the King Chulalongkorn Memorial Hospital, Bangkok, Thailand. Consecutive patients who were aged 18 or older and indicated for ERCP during July to October 2016 were screened. Exclusion criteria were pregnancy, American Society of Anesthesiology (ASA) physical status class III-IV, unstable vital signs or surgically altered anatomy, need to change the patient's position during the procedure, needing a specific ERCP position (such as hilar biliary obstruction and pancreatic pathology), and informed consent could not be obtained. The study protocol was approved by the Chulalongkorn University Institutional Review Board (IRB number 624/58).

\section{Fluoroscopy system and setting}

The mobile C-arm, under-couch fluoroscopy system (BV Pulsera, Philips, Amsterdam, The Netherlands), with the "last image hold" function producing pulsed fluoroscopy at 12.5 pulses per second, was used in this study. The examination mode of the anatomically programmed fluoroscopy was selected as the abdomen and the nominal II format was set at $31 \mathrm{~cm}$. Tube voltage and tube current-time were adjusted automatically to maintain a constant radiation dose entering the over-couch image intensifier. The lead curtain was mounted around the table during procedures. A well-trained assistant controlled the

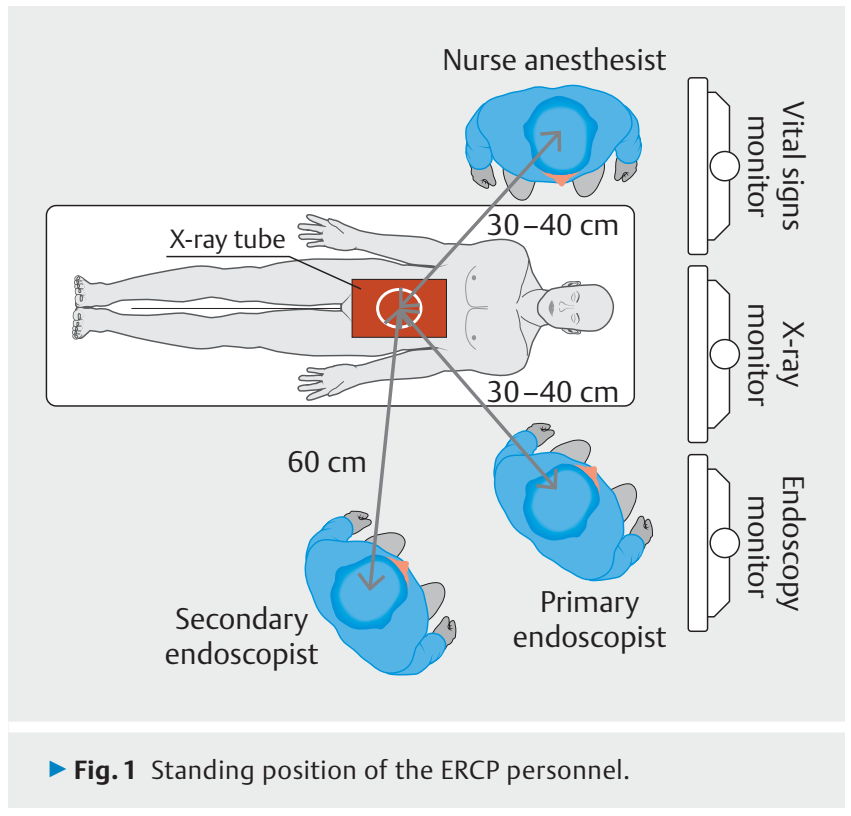

fluoroscopy according to the endoscopist's request but it was not adjusted for image magnification.

\section{Procedure and radiation measurement}

Patient age, gender, body mass index, body thickness, and indications for ERCP were recorded. Patients were randomized into two groups by computer-generated codes in block-of-four. Randomization codes were inserted in the sequentially numbered envelopes. An envelope was opened consecutively in the endoscopy room to assign the patient's position (prone or LLD). All ERCP personnel wore a wraparound lead apron and thyroid collar. ERCP was performed according to the indication in a standard technique as described elsewhere [15] under moderate sedation using intravenous meperidine and midazolam. The distance of all ERCP personnel from the $\mathrm{X}$-ray tube was approximately 30 to $40 \mathrm{~cm}$ for the primary endoscopist and the nurse anesthetist and $60 \mathrm{~cm}$ for the secondary endoscopist ( Fig. 1). The detector units, Personal Dose Meter (PDM) of the DoseAware system (Philips, Amsterdam, the Netherlands), were placed outside the thyroid collar of each ERCP team member on the side that was close to the fluoroscopy system; that is, on the left side of the primary and secondary endoscopists, and on the right side of the nurse anesthetist, and these represented the eye exposure of the involved personnel. The PDM was calibrated in terms of the dose equivalent quantity $H(p)$ (3) representing radiation doses at the ocular lens [16]. In this study, the primary endoscopist began first and the attending endoscopist replaced him whenever the ERCP procedure failed to progress. To maintain the correct PDM positions, the detector units were swapped between the two endoscopists whenever they swapped their positions. After the ERCP procedure, the total fluoroscopic time (minute), fluoroscopy tube voltage $(\mathrm{kV})$, fluoroscopy tube current $(\mathrm{mA})$, patient entrance skin dose rate $(\mathrm{mGy} / \mathrm{min})$, dose area product $\left(\mathrm{Gy}-\mathrm{cm}^{2}\right)$ and equivalent dose (mSv) were recorded. The ocular lens equivalent dose was presented as the equivalent dose per procedure (mSv/pro- 
cedure) and the equivalent dose per fluoroscopy time (equivalent dose rate; $\mathrm{mSv} /$ hour) [17].

\section{Sample size and statistical analysis}

A previous study [18] measured radiation exposures in 4 different areas of the endoscopist, including left eye, thyroid, left forearm and left leg, while performing ERCP on patients with prone and LLD positions. The sample size was calculated based on the data for ocular lens equivalent dose of the endoscopist in patients with prone and LLD positions $(0.059$ and $0.084 \mathrm{mSv}$, respectively) from a previous study [18]. To demonstrate a $20 \%$ difference in the ocular lens equivalent dose at a power of $90 \%$ and type I error of $5 \%$, the calculated number of needed patients in each group was 27 . Continuous variables were displayed as the mean \pm standard deviation (SD), or median (interquartile range, $[I Q R])$, and the difference between the two groups was analyzed with a Student's t-test, or Mann-Whitney $\mathrm{U}$ test where appropriate. Categorical variables were displayed as the percentage or proportion and the differences between the two groups were analyzed with a Chi-squared or Fisher's exact test where appropriate. Statistical analysis was performed with IBM SPSS statistics 19. A two-sided $P$ value $<0.05$ was considered to be significant.

\section{Results}

During the study period, there were 71 consecutive patients who underwent ERCP, and 16 patients were excluded because of a hilar lesion ( $n=14$ ) or unstable vital signs $(n=2)$. Fifty-five patients were randomized to prone $(n=27)$ and LLD $(n=28)$ position groups. One patient in the LLD group was excluded because the position was changed to prone during the procedure because of difficult cannulation and a double guidewire technique to achieve deep biliary cannulation was required. The final analysis was made from these 54 patients ( 27 patients in each group; Fig.2). Demographic parameters including age, gender, body mass index (BMI) in the prone position and LLD position groups were not different ( $>$ Table 1 ). Indications for ERCP in the prone and LLD groups were choledocholithiasis (63\% vs. $67 \%$ ), malignant biliary stricture (30\% vs. $22 \%$ ) and benign biliary stricture ( $7 \%$ vs. $11 \%$; $P=0.780$ ), respectively. The switch-over rate from primary to secondary endoscopist were $74 \%$ and $67 \%$ in prone and LLD groups, respectively ( $P=$ $0.766)$. The mean vertical thickness in the prone and LLD groups was 27.2 vs. $20.2 \mathrm{~cm}(P<0.001)$, respectively. Median fluoroscopy time, median fluoroscopy tube voltage, median fluoroscopy tube current, median dose area product, and median patient entrance skin dose rate in the prone and LLD groups were 4.14 vs. $4.06 \mathrm{~min}(P=0.993), 70$ vs. $72 \mathrm{kV}(P=0.549), 2.30$ vs. $2.29 \mathrm{~mA}(P=0.659), 23.2$ vs. $22.3 \mathrm{~Gy}-\mathrm{cm}^{2}(P=0.742)$, and 5.5 vs. $5.7 \mathrm{mGy} / \mathrm{min}(P=0.197)$, respectively ( $\triangleright$ Table 1$)$.

Median ocular lens equivalent doses in the primary endoscopist were significantly lower in the prone versus the LLD positions ( 0.0192 vs. $0.0307 \mathrm{mSv}, P=0.035)$ and the nurse anesthetist median ocular lens equivalent doses were also significantly different ( 0.0173 vs. $0.0442 \mathrm{mSv}, P=0.002)$, but the secondary endoscopist did not show a difference ( $\vee$ Table 2 ). Median ocu-

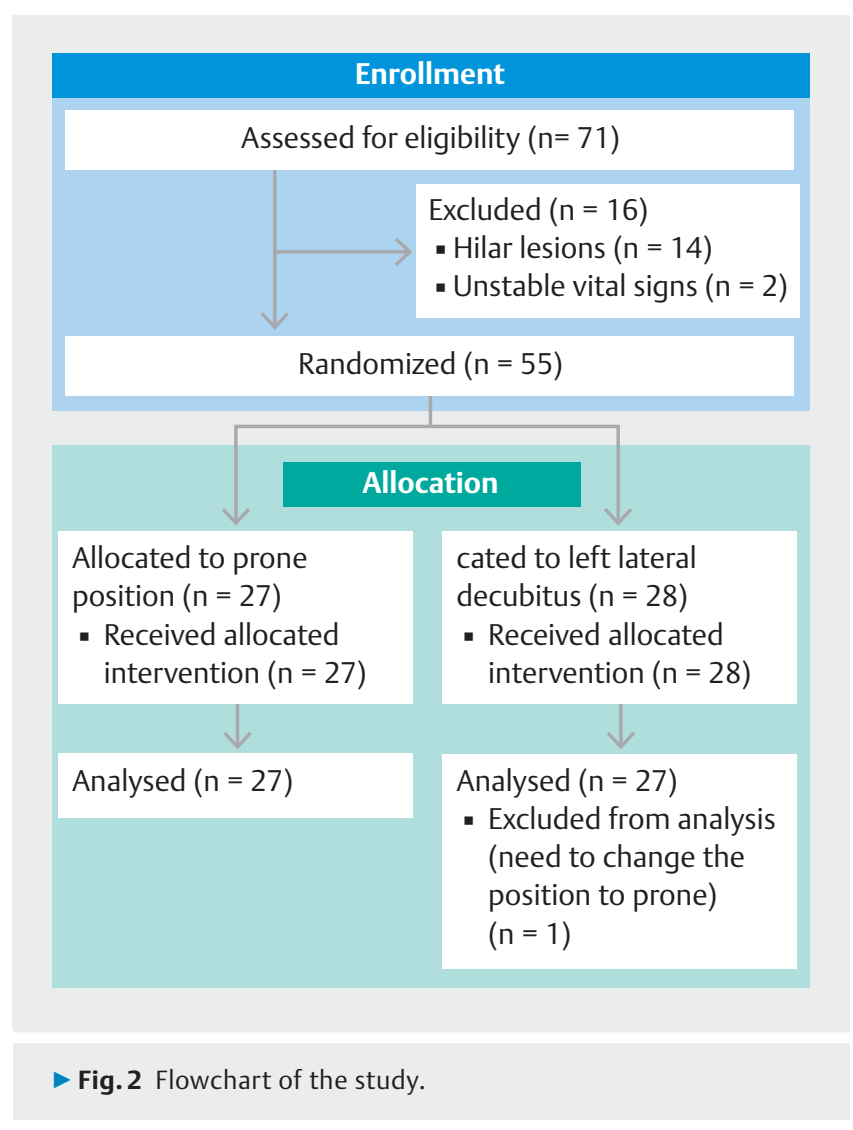

lar lens equivalent dose rates were significantly lower in the prone position than in the LLD position in all 3 personnel $(0.28$ vs. $0.43 \mathrm{mSv} / \mathrm{hr} ; P=0.001$ ) in the primary endoscopist, 0.18 vs. $0.25 \mathrm{mSv} / \mathrm{hr}(P=0.015)$ in the secondary endoscopist and 0.23 vs. $0.54 \mathrm{mSv} / \mathrm{hr}(P<0.001)$ in the nurse anesthetist ( $>$ Table 2$)$.

We then calculated the possible number of cases and fluoroscopy time allowances for each individual under the two positions. According to the new recommendation of the International Commission on Radiological Protection (ICRP), which limits the annual radiation dose for the ocular lens at $20 \mathrm{mSv}$ [2], the calculated maximum number of cases per annum for each staff member without wearing radiation protective eyewear in prone and LLD positions were 1,042 and 651 cases for the primary endoscopist, 2,083 and 1,302 cases for the secondary endoscopist, and 1,157 and 473 cases for the nurse anesthetist ( $\triangleright$ Table 3 ). The annual fluoroscopy time limit in the prone and LLD positions were 71.42 and 46.51 hours for the primary endoscopist, 111.11 and 80 hours for the secondary endoscopist, and 86.96 and 37.03 hours for the nurse anesthetist ( $\triangleright$ Table 3$)$.

\section{Discussion}

This study demonstrated that performing ERCP in a prone position significantly exposed the primary endoscopist and the nurse anesthetist to lower ocular lens equivalent doses. By simply changing the patient position from the LLD position to a prone position, the ocular lens equivalent doses to the primary 
- Table 1 Baseline characteristics and fluoroscopic parameters of patients in prone and left lateral decubitus (LLD) positions.

\begin{tabular}{|c|c|c|c|}
\hline Parameters & $\begin{array}{l}\text { Prone } \\
\mathrm{n}=\mathbf{2 7}\end{array}$ & $\begin{array}{l}\text { LLD } \\
n=27\end{array}$ & $P$ value \\
\hline Age $^{1}$ (years) & $67(15)$ & $65(27)$ & 0.723 \\
\hline Male:Female (n) & $15: 12$ & $10: 17$ & 0.17 \\
\hline $\mathrm{BMI} \mathrm{I}^{2}\left(\mathrm{~kg} / \mathrm{m}^{2}\right)$ & $22.2 \pm 4.07$ & $22.9 \pm 3.45$ & 0.476 \\
\hline \multicolumn{4}{|l|}{ Indication for ERCP (\%) } \\
\hline - Choledocholithiasis & 63 & 67 & 0.78 \\
\hline - Malignant biliary stricture & 30 & 22 & \\
\hline - Benign biliary stricture & 7 & 11 & \\
\hline Switch over from primary to secondary endoscopist n (\%) & $20(74)$ & $18(67)$ & 0.766 \\
\hline Vertical thickness $(\mathrm{cm})^{2}$ & $20.2 \pm 4.18$ & $27.2 \pm 3.71$ & $<0.001$ \\
\hline Fluoroscopy time ${ }^{1}$ (minutes) & $4.14(4)$ & $4.06(4)$ & 0.993 \\
\hline Fluoroscopy tube voltage ${ }^{1}(\mathrm{kV})$ & $70(12)$ & $72(7)$ & 0.549 \\
\hline Fluoroscopy tube current ${ }^{1}(\mathrm{~mA})$ & $2.30(0)$ & $2.29(0)$ & 0.659 \\
\hline $\begin{array}{l}\text { Dose area product }{ }^{1} \\
\left(G y-\mathrm{cm}^{2}\right)\end{array}$ & $23.2(19)$ & $22.3(24)$ & 0.742 \\
\hline Patient entrance skin dose rate $^{1}$ (mGy/min) & $5.5(1)$ & $5.7(4)$ & 0.197 \\
\hline $\begin{array}{l}\text { BMI, body mass index; ERCP, endoscopic retrograde cholangiopancreatography } \\
{ }^{1} \text { Data presented as the median (interquartile range; IQR) }\end{array}$ & & & \\
\hline
\end{tabular}

- Table 2 Ocular lens equivalent dose of ERCP personnel in prone and LLD positions.

\begin{tabular}{|c|c|c|c|c|c|c|}
\hline \multirow[t]{2}{*}{ ERCP personnel } & \multicolumn{2}{|c|}{$\begin{array}{l}\text { Median (IQR) ocular lens equivalent dose } \\
\text { (mSv) }\end{array}$} & \multirow[t]{2}{*}{$P$ value } & \multicolumn{2}{|c|}{$\begin{array}{l}\text { Median (IQR) ocular lens equivalent dose } \\
\text { rate (mSv/hour) }\end{array}$} & \multirow[t]{2}{*}{$P$ value } \\
\hline & Prone & LLD & & Prone & LLD & \\
\hline Primary endoscopist & $0.0192(0.0207)$ & $0.0307(0.0245)$ & 0.035 & $0.28(0.21)$ & $0.43(0.20)$ & 0.001 \\
\hline Secondary endoscopist & $0.0096(0.0135)$ & $0.0154(0.0192)$ & 0.113 & $0.18(0.09)$ & $0.25(0.12)$ & 0.015 \\
\hline Nurse anesthetist & $0.0173(0.0250)$ & $0.0422(0.0346)$ & 0.002 & $0.23(0.18)$ & $0.54(0.41)$ & $<0.001$ \\
\hline
\end{tabular}

- Table 3 Maximum annual procedures and fluoroscopy time for ERCP personnel (without wearing eye protection) not to exceed the ocular lens equivalent dose threshold of $20 \mathrm{mSv}$.

\begin{tabular}{|l|l|l|l|l|}
\hline \multirow{2}{*}{ ERCP personnel } & \multicolumn{2}{|c|}{ Maximum procedures } & \multicolumn{2}{l|}{ Maximum annual fluoroscopy time (hours) } \\
\cline { 2 - 5 } & Prone & LLD & Prone & LLD \\
\hline Primary endoscopist & 1,042 & 651 & 71.42 & 46.51 \\
\hline Secondary endoscopist & 2,083 & 1,302 & 111.11 & 80 \\
\hline Nurse anesthetist & 1,157 & 473 & 86.96 & 37.03 \\
\hline ERCP, endoscopic retrograde cholangiopancreatography; LLD, left lateral decubitus & & \\
\end{tabular}

endoscopist and the nurse anesthetist were reduced by $37.5 \%$ and $59.0 \%$, respectively. Although the equivalent dose in the secondary endoscopist was also reduced by $37.5 \%$, this did not reach a statistically significant difference. We speculate that the sample size was too small to have enough power to demonstrate the difference in equivalent dose to the secondary endoscopist between the two positions. Of note, the secondary endoscopist is exposed to radiation at a much lower level than 
the other two personnel. Interestingly, when we calculated the ocular lens equivalent dose per the procedure time (dose rate) [17], the prone position significantly yielded the lower dose rates in all three personnel when compared with the LLD position. This calculation eliminated variation in fluoroscopy time, which was influenced by procedure difficulty [19]. This confirmed that, within the same timeframe, the prone position significantly lowered the ocular-radiation exposure to all personnel.

As we hypothesized earlier, the two factors that might affect the radiation exposure to ERCP personnel were modulated dose of voltage adjustment by the X-ray tube and the scattered radiation acquired from the increase in distance between the image intensifier and the patient [11,20]. Among all baseline characteristics, we showed that only the vertical thickness of the patient in LLD group was significantly higher than in the prone position group. Interestingly, radiation doses from the $X$-ray tube, called the modulator effect (tube voltage and tube current), were not significantly different. Furthermore, the dose area product and the patients' skin dose rates were also not significantly different. These reflected that the fluoroscopy system did not significantly increase the tube voltage and that the patient was not exposed to more radiation from the voltage adjustment when changing the position from prone to LLD. Unlike the previous study in a phantom, which demonstrated that the radiation dose significantly increased along with the increment of thickness [11], the difference in the phantom was that the density of the medium is more homogeneous than the real human body and this, in turn, can cause the difference in radiation penetrance [21]. The current study demonstrated that, in a real human body, the increment of vertical thickness from $20 \mathrm{~cm}$ to $27 \mathrm{~cm}$ did not significantly increase the exposure by the X-ray tube. Perhaps the scattered ray from the patients is the only thing responsible for the increase in radiation exposure to the ERCP personnel. Therefore, to reduce radiation scatter during ERCP, the image intensifier should be positioned as close to the patient's body as possible [5].

The ocular lens is composed of radiosensitive tissues that are at risk of developing cataracts after receiving significant ionizing radiation [22]. Since April 2011, the ICRP has lowered the equivalent dose limit for the lens of the eyes during occupational exposure from $150 \mathrm{mSv} /$ year to $20 \mathrm{mSv} /$ year as averaged over the period of 5 years, with no single year exceeding $50 \mathrm{mSv}$ [2]. However, radiation protection for eyes has not yet been mandated by major international guidelines on radiation protection during ERCP. Radiation-protective eyewear is recommended as an optional measure by the American Society for Gastrointestinal Endoscopy (ASGE) [23] and is recommended only when using over-couch fluoroscopy by the European Society of Gastrointestinal Endoscopy (ESGE) [5]. Patient position in ERCP can be either prone, supine, or LLD. Selection of position is dependent upon patient factors (e.g., neck mobility, presence of abdominal drains or wounds), airway management [24], or endoscopist's preference. LLD is considered to be easier on airway management and scope intubation/positioning; however, the examination is limited for extra-hepatic bile duct indications, because the anatomical orientation is suboptimal for pancreatic duct or biliary bifurcation [24].

Although LLD is not a common position for ERCP in the United States [12], in Thailand, LLD is the most common position, which accounts for $50 \%$, followed by prone $(32.7 \%)$ and then supine (17.3\%; personal unpublished survey from ERCP endoscopists across Thailand). Furthermore, many endoscopists have overlooked radiation protection to their eyes. In the same survey, only $38.2 \%$ reported availability of radiation-protective eyewear and only $7.5 \%$ reported wearing that eyewear at all times. Likewise, the survey from Korea [25] revealed that radiation-protective eyewear was used by endoscopists only $37.8 \%$ of the time, while a lead apron and thyroid shield were used $98.7 \%$ and $94.7 \%$ of the time, respectively.

This study emphasized that not only the primary endoscopist but also the nurse anesthetist is at risk of developing cataracts and eyewear use should be the standard of practice because of the potential for exceeding the allowance of annual radiation exposure to the ocular lens [2] (if the annual radiation exposure exceeds 1,000 procedures in a prone position or 600 procedures in an LLD position). The calculated procedure limit was based on the C-arm fluoroscopy system, and the ALARA approach applied in this study, for example using pulsed fluoroscopy, had the lowest possible pulsed rate, rather than continuous fluoroscopy, stored as the "last image hold" rather than taking radiographs, and avoidance of the magnification mode [5]. When the ALARA protocol is not in effect or when facing a complex ERCP case that requires a longer fluoroscopy time, the limited number of procedures per annum could have been lower.

This study had some limitations. First, we could not blind the endoscopist and the assistant who controlled the fluoroscopy. However, the attending staff members had experience in ERCP of more than 200 cases/year and were well trained on radiation safety and complied with the ALARA principle. Because the results of fluoroscopy parameters, especially fluoroscopy times, were no different between the two groups and were comparable with other studies $[26,27]$, there was low bias for the fluoroscopy control in this study. Second, we excluded complex cases, especially hilar cholangiocarcinoma, because of the need for an antero-posterior view of fluoroscopy, as those indications might require a longer fluoroscopy time and result in greater radiation exposure $[5,28]$. We then calculated an equivalent dose rate to eliminate variation in fluoroscopy time and this might be appropriate for radiation monitoring rather than the mean dose per procedure [17]. Lastly, because it was not practical to place the PDM near the eyes as that might obscure the visual field of the personnel, the ocular lens doses were calculated based on calibration from the measured doses at the neck level. This adjustment was suggested by the previous study that demonstrated that placement of the PDMat the thyroid collar was suitable for the ocular lens dose assessment when compared with direct measurement close to the eyes [20]. Furthermore, the ocular lens doses in the current study were in line with the previous study that made measurements directly between the eyes [17]. Regarding the involvement of trainees, the switch-over rate from primary to second- 
ary endoscopist was comparable in both groups and we always swapped the PDMs when the primary and secondary endoscopists changed their positions. This ensured that the correct exposure measurement was based on the standing position (not based on the individual). Of note, we observed that the trainees spent most of their time on biliary cannulation but that would not have much of an effect on the radiation exposure because it required proportionally less fluoroscopy.

\section{Conclusion}

In conclusion, performing ERCP with a patient in a prone position with the image intensifier positioned as close as possible to the patient body and using a lead curtain reduces by one-third the ocular-radiation exposure to ERCP personnel from the LLD position. Therefore, more annual ERCPs can be performed in patients in the prone position under the recommended dose limit.

\section{Acknowledgements}

This research was supported by a grant for International Research Integration: Chula Research Scholar, Rachadaphiseksomphot Endowment Fund (2300052001).

\section{Competing interests}

None

References

[1] Campbell N, Sparrow K, Fortier M et al. Practical radiation safety and protection for the endoscopist during ERCP. Gastrointest Endosc 2002; 55: $552-557$

[2] Stewart FA, Akleyev AV, Hauer-Jensen M et al. ICRP publication 118: ICRP statement on tissue reactions and early and late effects of radiation in normal tissues and organs-threshold doses for tissue reactions in a radiation protection context. Ann ICRP 2012; 41: 1-322

[3] Mehta K. Radiation: basic principles. J Vasc Surg 2005; 42: 1237 1238

[4] [Anonymous] The 2007 Recommendations of the International Commission on Radiological Protection. ICRP publication 103. Ann ICRP 2007; 37: 1-332

[5] Dumonceau JM, Garcia-Fernandez FJ, Verdun FR et al. Radiation protection in digestive endoscopy: European Society of Digestive Endoscopy (ESGE) guideline. Endoscopy 2012; 44: 408-421

[6] Strauss KJ, Kaste SC. The ALARA (as low as reasonably achievable) concept in pediatric interventional and fluoroscopic imaging: striving to keep radiation doses as low as possible during fluoroscopy of pediatric patients - a white paper executive summary. Pediatr Radiol 2006; 36: (Suppl. 02): 110-112

[7] Buls N, Pages J, Mana F et al. Patient and staff exposure during endoscopic retrograde cholangiopancreatography. $\mathrm{Br}$ J Radiol 2002; 75: $435-443$

[8] Cohen G, Brodmerkel G] Jr, Lynn S. Absorbed doses to patients and personnel from endoscopic retrograde cholangiopancreatographic (ERCP) examinations. Radiology 1979; 130: 773 - 775
[9] Killewich LA, Falls G, Mastracci TM et al. Factors affecting radiation injury. J Vasc Surg 2011; 53: 9S - 14 S

[10] Vano E, Gonzalez L, Fernandez JM et al. Influence of patient thickness and operation modes on occupational and patient radiation doses in interventional cardiology. Radiat Prot Dosimetry 2006; 118: 325 330

[11] Hadjiconstanti AC, Messaris GA, Thomopoulos KC et al. Optimisation of patient dose and image quality in endoscopic retrograde cholangiopancreatography: a phantom-based evaluation. Radiat Prot Dosimetry 2017; 175: $118-123$

[12] Ferreira LE, Baron TH. Comparison of safety and efficacy of ERCP performed with the patient in supine and prone positions. Gastrointest Endosc 2008; 67: 1037 - 1043

[13] Terruzzi V, Radaelli F, Meucci G et al. Is the supine position as safe and effective as the prone position for endoscopic retrograde cholangiopancreatography? A prospective randomized study Endoscopy 2005; 37: $1211-1214$

[14] Batheja M, Harrison ME, Das A et al. Optimal positioning for ERCP: efficacy and safety of ERCP in prone versus left lateral decubitus position. ISRN Endoscopy 2013; 2013: 1 - 6

[15] [Anonymous] ERCP. 2nd ed. Philadelphia: Saunders Elsevier; 2013

[16] Martin C]. Personal dosimetry for interventional operators: when and how should monitoring be done? $\mathrm{Br}$ J Radiol 2011; 84: 639-648

[17] Garg MS, Patel P, Blackwood M et al. Ocular radiation threshold projection based off of fluoroscopy time during ERCP. Am J Gastroenterol 2017; 112: $716-721$

[18] Ridtitid W, Suwanboonrit W, Rerknimitr R et al. Difference in radiation exposures to patient and endoscopist between performing ERCP in patients lying prone and lying left lateral decubitus. Gastrointest Endosc 2011; 73: AB411-412

[19] Churrango G, Deutsch JK, Dinneen HS et al. Minimizing radiation exposure during ercp by avoiding live or continuous fluoroscopy. J Clin Gastroenterol 2015; 49: e96-e100

[20] Principi S, Ginjaume M, Duch MA et al. Influence of dosemeter position for the assessment of eye lens dose during interventional cardiology. Radiat Prot Dosimetry 2015; 164: 79-83

[21] Gurjar OP, Mishra SP, Bhandari V et al. Radiation dose verification using real tissue phantom in modern radiotherapy techniques. J Medical Physics 2014; 39: 44-49

[22] Rehani MM, Vano E, Ciraj-Bjelac O et al. Radiation and cataract. Radia Prot Dosimetry 2011; 147: $300-304$

[23] Committee AT, Pedrosa MC, Farraye FA et al. Minimizing occupational hazards in endoscopy: personal protective equipment, radiation safety, and ergonomics. Gastrointest Endosc 2010; 72: 227 - 235

[24] Maple JT. Preparation for ERCP. In: Baron TH, Kozarek RA, Carr-Locke DL (eds). ERCP. 2nd ed. Philadelphia: Elsevier Saunders; 2013

[25] Shin JM, Lee TH, Park SH et al. A survey of the radiation exposure protection of health care providers during endoscopic retrograde cholangiopancreatography in Korea. Gut Liver 2013; 7: 100 - 105

[26] Kachaamy T, Harrison E, Pannala R et al. Measures of patient radiation exposure during endoscopic retrograde cholangiography: beyond fluoroscopy time. World J Gastroenterol 2015; 21: 1900-1906

[27] Muniraj T, Aslanian HR, Laine L et al. A double-blind, randomized, sham-controlled trial of the effect of a radiation-attenuating drape on radiation exposure to endoscopy staff during ERCP. Am J Gastroenterol 2015; 110: 690-696

[28] Alkhatib AA, Abdel Jalil AA, Faigel DO et al. Anatomical location of pathology is predictive of prolonged fluoroscopy time during ERCP: a multicenter American study. Digest Dis Sci 2015; 60: 1787-1792 